\title{
Angular filtering by Bragg photonic microstructures fabricated by physical vapour deposition
}

\author{
Lina Grineviciute ${ }^{1}$, Ceren Babayigit ${ }^{2}$, Darius Gailevičius ${ }^{3,4^{*}}$, Emre Bor ${ }^{2,5}$, Mirbek Turduev ${ }^{5}$, Vytautas \\ Purlys $^{3,4}$, Tomas Tolenis ${ }^{1}$, Hamza Kurt ${ }^{2}$, Kestutis Staliunas ${ }^{6,7}$ \\ ${ }^{1}$ Center for Physical Sciences and Technology, Savanoriu Ave. 231, LT-02300 Vilnius, Lithuania \\ ${ }^{2}$ Department of Electrical and Electronic Engineering, TOBB University of Economics and Technology, \\ Söğütözü Str. 43, 06510, Ankara, Turkey \\ ${ }^{3}$ Vilnius University, Faculty of Physics, Laser Research Center, Sauletekio Ave. 10, Vilnius, Lithuania \\ ${ }^{4}$ Femtika LTD, Sauletekio Ave. 15, LT-10224, Vilnius, Lithuania \\ ${ }^{5}$ Department of Electrical and Electronic Engineering, TED University, Ziya Gökalp Str. 48, \\ 06420, Ankara, Turkey \\ ${ }^{6}$ Institució Catalana de Recerca i Estudis Avancats (ICREA), Passeig Lluís Companys 23, 08010, \\ Barcelona, Spain \\ ${ }^{7}$ Universitat Politècnica de Catalunya (UPC), Rambla Sant Nebridi 22, 08222, Terrassa (Barcelona) Spain \\ *Corresponding author: darius.gailevicius@ff.stud.vu.lt
}

Keywords: Spatial filtering; Photonic crystals; Physical vapour deposition; Glancing angle deposition; FDTD; Surface grating.

\begin{abstract}
We propose and experimentally demonstrate spatial filtering by photonic crystals in a Bragg configuration. Compared to the Laue configuration, where spatial filtering was already demonstrated before, the Bragg configuration is more technologically challenging, as the longitudinal periods of such structures must be shorter than the operational wavelength. The proposed configuration is designed and analyzed by FDTD simulations and the multilayer structure is fabricated by physical vapour deposition on the microstructured substrate. The measurements of the angle/wavelength transmission of the fabricated structure show the signatures of the angular filtering.
\end{abstract}

\section{Introduction}

The idea of the angular/spatial filtering by photonic crystals (PhCs) relies on selective diffraction of light from the double-periodic photonic structures. The angular components of the incident light at resonance with the transverse and longitudinal periodicities of the structure diffract efficiently and they are removed from the zero-diffraction order of the transmitted beam. On the other hand, the angular components that are out of the resonance propagate unaffected or weakly affected by the photonic structure. Therefore, the angular/spatial filtering (low-angle-pass or high-angle-pass filtering) can be obtained by a proper interplay between the longitudinal and transverse periodicities, i.e., by a suitable geometry of the PhCs.

The spatial filtering by the PhCs was theoretically predicted [1-3] and experimentally demonstrated [4,5]. In particular, the PhCs filtering has been already implemented for intracavity angular filtering in microchip lasers, where the beam spatial quality was substantially improved and the brightness of the emission was increased [6]. Due to small dimensions, mechanical stability, and no need for focusing or additional optical elements, 
such $\mathrm{PhC}$ filtering shows itself as an attractive alternative solution for conventional techniques, where the filtering is achieved by a confocal arrangement of lenses with the filtering diaphragm in a focal plane.

On the other hand, apart from conventional filtering, there have been attempts to apply more sophisticated schemes for the light manipulation, in particular by using passive [7,8] or light-induced [9-11] Bragg gratings. However, the latter approach uses an additional laser source. To the best of author's knowledge, the filtering was demonstrated only using cold gases, which limits its applicability in the microoptical systems. Population density gratings can also be induced by using optical pulses in a nonlinear medium that does not require an additional laser source [12]. These alternative methods, however, require complex designs and additional optical components that limit their applications, especially in the microlaser systems. On the contrary, the PhCs based angular filters are extremely compact. Hence, they are especially promising solutions for the intracavity use in the microlasers.

The PhC filters so far were designed and fabricated to work in Laue diffraction regime where the waves diffract predominantly in a forward direction as schematically illustrated in Fig.1(a). In general, the Laue regime occurs for $d_{\mathrm{z}}>\lambda$. In this case, no full photonic band gap exists. As the wave propagates through the $\mathrm{PhC}$ in Laue regime, the periodic revivals called Rabi oscillations occur between the zero- and first- diffracted orders (Fig. 1 (d)). For this reason, the length of the structure must be selected precisely for to get efficient filtering. The advantage of the Laue regime is that the lattice periods are well achievable by optical fabrication techniques in organic/inorganic materials [13-15], as the $\mathrm{PhC}$ periods along the optical axis $d_{\mathrm{z}}$ are in the range of tens of microns, and along the transverse directions $d_{\mathrm{x}, \mathrm{y}}$ - of several microns.

The spatial filtering is also possible in the so-called Bragg regime (Fig.1(b)). Here the length of the structure is less critical, as the Rabi oscillations between filtered and unfiltered modes do not appear. and, instead, exponential decay of the filtered modes occur (Fig.1(e)). In the Bragg regime $d_{\mathrm{z}}<\lambda$ (most typically $d_{\mathrm{z}} \approx \lambda / 2$ ), the optical fabrication techniques can be hardly implemented. Holographic methods, despite promising experiments [16,17], require ultraviolet sources and complicated optical designs. Therefore, their use for the PhC fabrication is very restricted, especially when the two-dimensional (2D)/three-dimensional (3D) volume gratings are required. Here, the physical vapour deposition method can be considered as a promising alternative solution for fabrication of such spatially filtering photonic structures in Bragg regimes. The thin film deposition methods have been demonstrated to be able to control the thickness and optical parameters at high accuracy [18]. Additionally, evaporation techniques, equipped with glancing angle deposition method, showed promising results in forming microstructures on structured substrates [19].

Other approaches of spatial filtering by photonic microstructures include the use of resonant gratings [20], 1D and 2D PhCs [21-24], graded index PhCs [25], metallic grids [26] and bi-dimensional doubly periodic gratings [27]. However, all these approaches are still lacking reasonable technological implementations. 

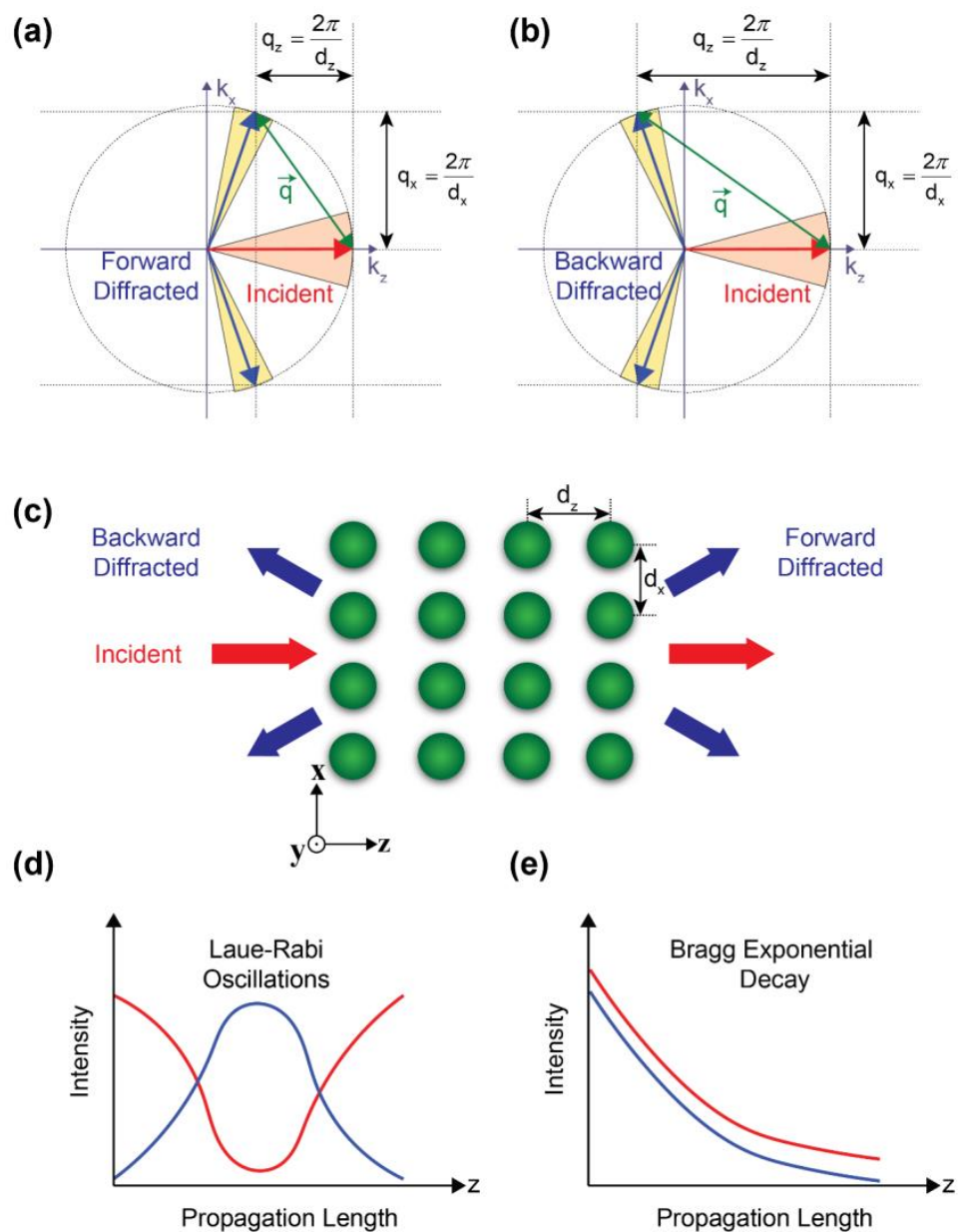

(e)

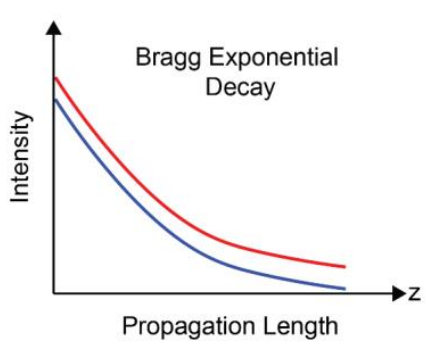

Fig.1. The principles of PhC angular filtering in (a) Laue and (b) Bragg configurations. The incident wave, with the wavevector indicated by red arrow $\left(k_{\mathrm{x}}, k_{\mathrm{z}}\right)=\left(0, k_{0}\right)$, diffracts from the double-periodic structure in (c) with the wavevectors $\left(q_{\mathrm{x}}, q_{\mathrm{z}}\right)$. The diffraction is efficient if the grating is at resonance, meaning that the wavevectors of the diffracted wave (blue arrows) are on the light-circle (or light-sphere in 3D). The diffracted waves in Laue configuration propagate in a forward direction and result in periodic energy exchange between the zero and first diffraction orders (d), whereas the waves in a Bragg configuration diffract backward, and thus result in an exponential decay (and correspondingly the stop-bands) of the incident wave (e).

The figure illustrates the high-angle-pass filter for simplicity, however evidently, a variation of the periods or wavelength results in a low-angle-pass filter.

In the presented study, we provide an experimental demonstration of the signatures of angular filtering in the Bragg configuration for the first time. We fabricate the PhCs on micro-textured substrates using physical vapour deposition method. The results experimentally prove the concept of spatial filtering but they do not lead to the ideal filtering performance yet, wherein the filtered waves are $100 \%$ attenuated. The main reason for that has become apparent in the process of the theoretical/experimental studies and is basically caused by too large transverse periods, and too small depth of the structures (too few longitudinal periods). Therefore, in concluding part of the article, we performed the finite-difference time-domain (FDTD) calculations with "improved" geometrical parameters and showed that the presented angular filtering principle works 
and leads to enhanced spatial filtering that can be applied for selected practical applications. However, the designed structure with enhanced geometrical properties was beyond the available fabrication facilities.

The article is organized in the following way: after the derivation of the Bragg band-gap conditions, we present the FDTD numerical simulation results of the light propagation through the structure. For the proper understanding of the experimental results, we calculated three versions of multilayered $\mathrm{PhC}$ structures for spatial filtering. The first version is based on a harmonically modulated substrate profile, and the second one is based on a blazed grating (triangular) substrate. Both versions can be realized by conformal dielectric coatings on a substrate with a periodic surface profile. The ideal case for spatial filtering would be a harmonic (wavy) one, yet the structure with a sufficient modulation depth and a smallest available period was the blazed one. Moreover, the real fabricated structure when starting from a blazed surface grating develops a smoother, almost sinusoidal character towards the outer layers. Therefore, the final result is a combination of the both, blazed and sinusoidal modulated versions. In this regard, the blazed-rounded structure was also calculated.

\section{Positions of the Band-Gaps}

The conditions for the Bragg bandgaps can be derived from the resonant scattering condition by using the simple geometric approach. All waves must satisfy the momentum and energy conservation laws. According to the latter, the wave with the wave-vector $\vec{k}_{0}=\left(k_{0, x}, k_{0, z}\right)$, and modulus $\left|\vec{k}_{0}\right|=\omega / c$ after diffracting in a periodic structure with reciprocal lattice vector $\vec{q}=\left(q_{x}, q_{z}\right)$, results into a diffracted wave with $\vec{k}=\vec{k}_{0}+\vec{q}$. The components of the lattice vector are $q_{x, z}=2 \pi / d_{x, z}$ (see Fig.1. for the definition of the transverse/longitudinal periods $d_{x, z}$ ). For efficient diffraction, the new wave with wave-vector $\vec{k}$ must satisfy the energy conservation law too, i.e., must satisfy $|\vec{k}|=$ $\omega / c=\left|\vec{k}_{0}\right|$. This means that in isotropic medium the initial as well as the final wavevectors must lie on the "light ring": $|\vec{k}|^{2}=(\omega / c)^{2}$, as illustrated in Figs. 1(a) and 1(b). As a result, a simple geometrical consideration allows us calculating the angles at which the Bragg diffraction is most efficient.

An incident wave propagating at the angle $\theta_{i}$ to the optical axis $\vec{k}_{0}=\left(k_{0} \sin \left(\theta_{i}\right), k_{0} \cos \left(\theta_{i}\right)\right)$, diffracting on a grating with $\vec{q}=\left(q_{x}, q_{z}\right)$, results in diffracted wave $\vec{k}=\vec{k}_{0}+\vec{q}=\left(k_{0} \sin \left(\theta_{i}\right)+q_{x}, k_{0} \cos \left(\theta_{i}\right)-q_{z}\right)$. The resonance condition $|\vec{k}|=\left|\vec{k}_{0}\right|$ then leads to the following implicit condition for the Bragg diffraction angle:

$$
|\vec{k}|=\frac{q_{x}^{2}+q_{z}^{2}}{2 q_{z} \cos \left(\theta_{i}\right)-2 q_{x} \sin \left(\theta_{i}\right)} .
$$

The Eq. (1) is a convenient tool to interpret the angular diffraction map, i.e., the dependence of diffracted wavelength on the incidence angle of $\theta_{i}$. It shows one Bragg 
diffraction curve in diffraction map, i.e., the diffraction due to the main harmonics $\vec{q}=\left(q_{x}, q_{z}\right)$ of the periodic structure. Considering that the gratings have different harmonics of periodicity: $\vec{q}_{n, m}=\left(m_{x} q_{x}, m_{z} q_{z}\right)$, different Bragg-diffraction branches appear:

$$
|\vec{k}|_{m x, m z}=\frac{m_{x}^{2} q_{x}^{2}+m_{z}^{2} q_{z}^{2}}{2 m_{z} q_{z} \cos \left(\theta_{i}\right)-2 m_{x} q_{x} \sin \left(\theta_{i}\right)} .
$$

Here $m_{x}=\cdots,-2,-1,0,1,2, \ldots$ corresponds to the diffraction order in the transverse direction, and $m_{z}=1,2, \ldots$ corresponds to the harmonics of longitudinal periodicity. The Eq. (2) bears an orientational character, showing the qualitative tendencies in the diffraction map. In order to quantitatively compare the precise FDTD calculations with the experimental results, the Eq. (2) is calibrated by the averaged refraction indices of the environments. Corresponding results are given in the following sections.

\section{Design approach and numerical results}

The design of multilayered $\mathrm{PhC}$ structure for spatial filtering is schematically represented in Fig. 2. The structure consists of alternating multilayers on a substrate where the interfaces of the layers are modified as a periodic array of sinusoidal wavy curves. It is important to note that for efficient spatial filtering the photonic structure must be periodically modulated in both longitudinal and transverse directions. Therefore, due to the wavy surface of the substrate, the modulation of the refractive index occurs not only in vertical but also in horizontal direction, resulting in a 2D PhC structure. Refractive indices of the high $(\mathrm{H})$, low (L) index layers and substrate (Sub) are $n_{H}=1.42, n_{L}=1.33$ and $n_{\text {Sub }}=1.5$, respectively. The thicknesses of the high and low refractive index layers are both equal to $d_{z} / 2=120 \mathrm{~nm}$, where the $d_{z}$ corresponds to the longitudinal period of the structure. The transverse lattice constant (period of wavy curves) is fixed to $d_{x}=1.67 \mu \mathrm{m}$ and number of layers is equal to $N_{L}=33$.
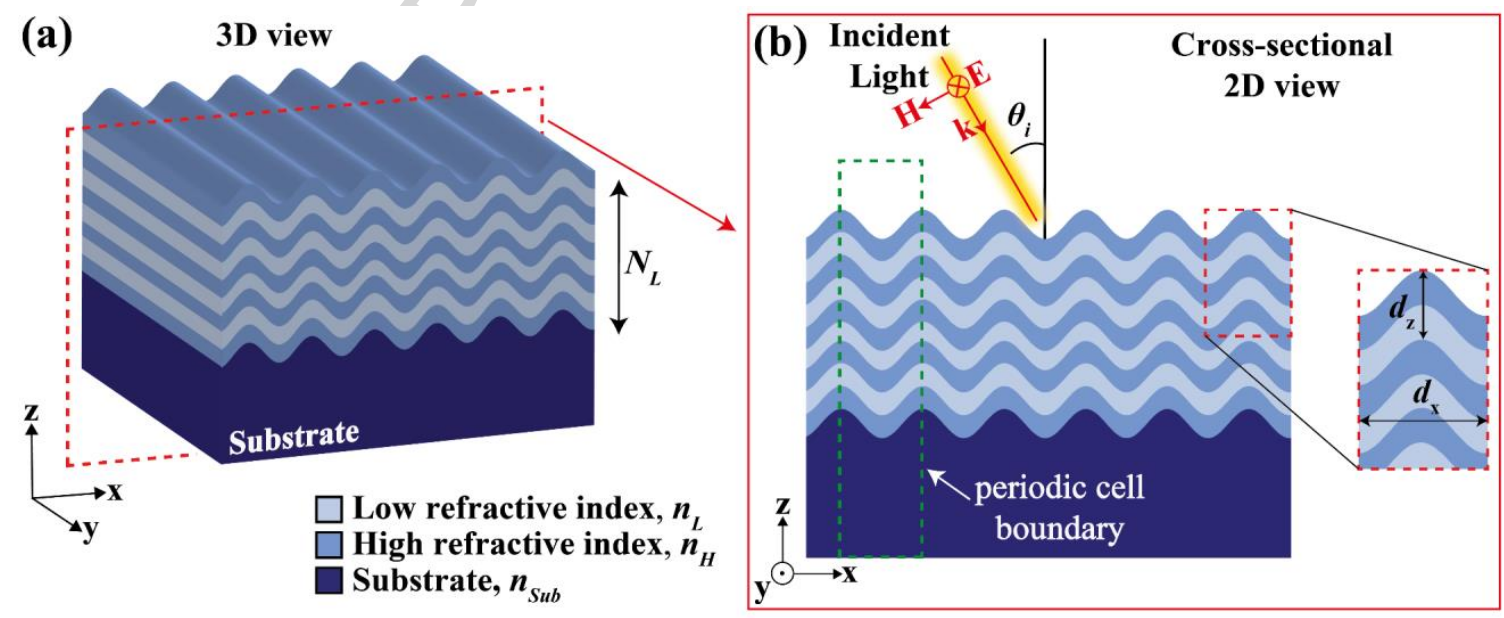

Figure 2. Schematic representation of the design approach. (a) $3 D$ view and (b) $2 D$ crosssectional view. Number of layers of the structure is represented by $N_{L}, d_{z}$ corresponds to the 
vertical period and $d_{x}$ denotes the horizontal period. The structure is illuminated with obliquely incidence source, and the incidence angle is represented by $\theta_{i}$.

The commercial FDTD software was used for the light-matter interaction analysis in the designed structure $[28,29]$. Due to the periodicity of the structure in the $x$-direction, time domain calculations were conducted via the supercell approach by assigning periodic boundary conditions in the $x$-direction. The dashed green frame in Fig. 2(b) shows the supercell boundaries. Here, the FDTD method solves the Maxwell's equations by infinitely repeating the defined periodical supercell in the $x$-direction. In order to observe the angular filtering property, the proposed structure is illuminated with $P$-polarized plane wave source at a variable angle $\left(-40^{\circ}<\theta_{i}<40^{\circ}\right)$ and the corresponding zero-order transmission is calculated in the range of the wavelengths between $450 \mathrm{~nm}$ and $850 \mathrm{~nm}$. Note that if radiation from a wide spectral bandwidth source is incident at an oblique angle, a phase shift occurs in the frequency domain [30]. Hence, to obtain the wideband response with an oblique incident plane wave, the Broadband Fixed Angle Source Technique (BFAST) is used in the supercell calculations [31]. The calculated transmission efficiencies evidencing the angular dependence are given as a map in Fig. 3(a).

As mentioned above, the fabrication of the layered structure was initiated on the blazed grating substrate. By considering the fabrication process, we first analyzed a blazed layered structure of the same periods. The blazing angle was adjusted as $10^{\circ}$ and the obtained transmittance map is given in Fig. 3(b). However, in the corresponding map, the angular filtering profile was broader in comparison with the presented map for wavy structure in Fig. 3(a). Next the blazed-rounded structure was calculated, Fig. 3(c). Here, one can see the obtained transmittance wave preserves the characteristics of the sinusoidal and blazed structures. Furthermore, for all cases, a cross-sectional profile of the intensity transmittance maps is extracted at $\lambda=582 \mathrm{~nm}$ (denoted by black solid line). The obtained results are given for sinusoidal, blazed and blazed-rounded layered structures in Figs. 3(d), 3(e) and 3(f), respectively. For the sinusoidal wavy structure, at zero incidence angle, we obtain transmission efficiency as high as $96 \%$, and filtering bands are present for angles at $\theta_{i}=-10^{\circ}$ and $10^{\circ}$, where transmission efficiency is decreased to $62 \%$. However, for the blazed case, there is no sign of explicit filtering between $-10^{\circ}$ and $10^{\circ}$ degrees. For the blazed-rounded structure, there is an asymmetric behavior as expected. Nevertheless, the filtering characteristic angles at $\theta_{i}=-10^{\circ}$ and $10^{\circ}$ are preserved.

In order to quantitatively compare FDTD-calculated Bragg-diffraction branches with analytical predictions, as stated previously, we calibrated Eq. 2 by the averaged refraction indices of the photonic structure. Obtained results are superimposed with transmission maps for both wavy and blazed structures in Figs. 3(a) and 3(b), respectively. Analytically calculated Bragg-diffraction branches are denoted by colored dashed lines on the transmission maps, showing good agreement with FDTD results. 


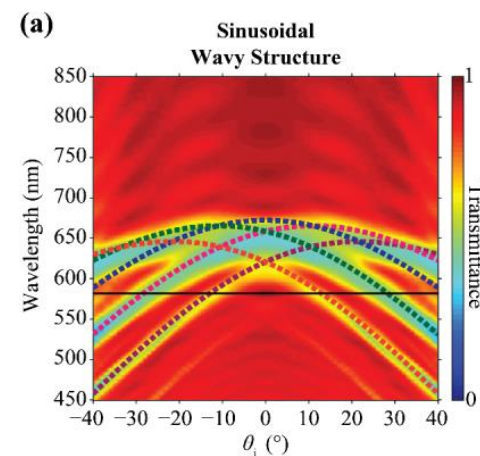

(d)

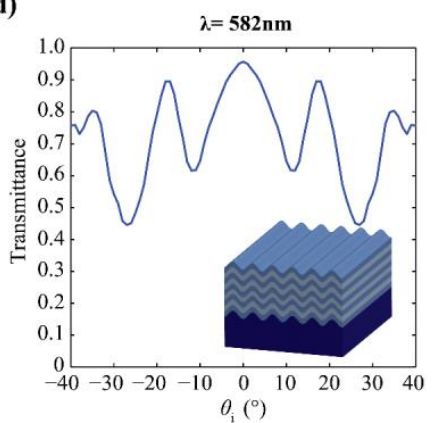

(b)

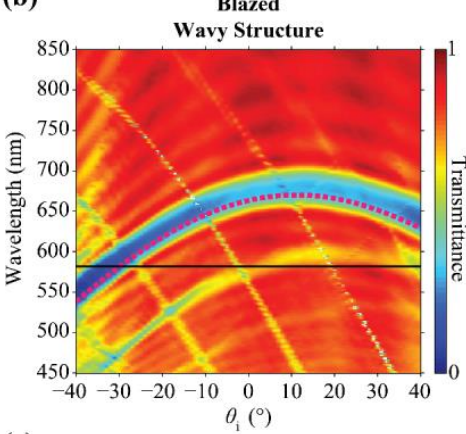

(e)

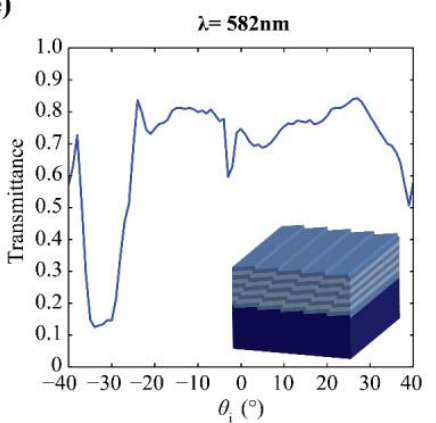

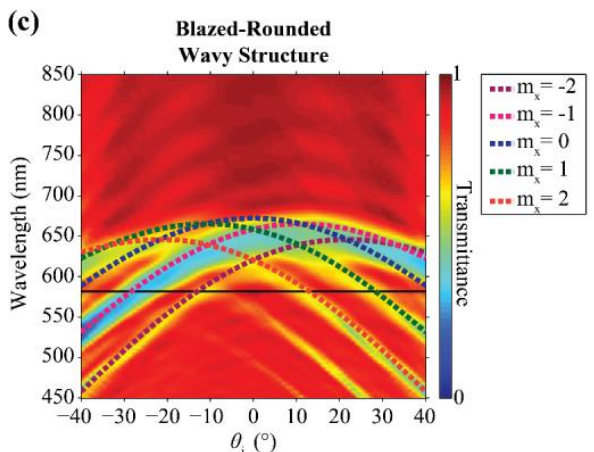

(f)

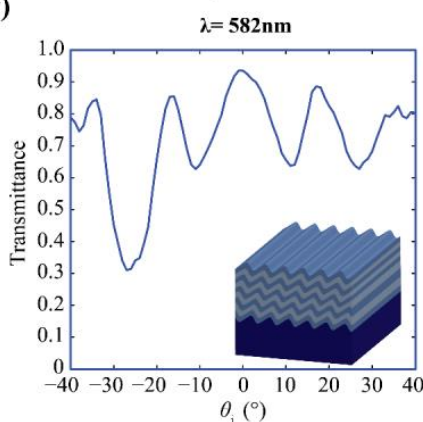

Figure 3. Zero-order calculated transmittance maps for incidence angle $\theta_{i}$ and wavelength $\lambda$ for structures with $d_{z}=240 \mathrm{~nm}$ and $d_{x}=1.67 \mu \mathrm{m}$ for (a) sinusoidal, (b) blazed and (c) blazedrounded wavy layered structures. Analytically calculated Bragg-diffraction branches are indicated by dashed curves. Cross-sectional profiles are shown for the wavelength $\lambda=582 \mathrm{~nm}$ for (d) sinusoidal, (e) blazed and (f) blazed-rounded wavy layered structures. The black solid line indicates the wavelength of interest. Schematically representations of the sinusoidal and blazed wavy layered structures are given as insets in the figure plots.

\section{Experimental study}

\subsection{Fabrication and measurement}

The fabrication of the designed structure involved three main steps. First, a PDMS mold of a commercially available diffraction grating (with a pitch of $d_{x}=1.67 \mu \mathrm{m}$ ) was made [32]. Second, a base grating was stamped on a soda-lime glass slides using a UV curable hybrid polymer (OrmoComp, $n_{\text {ref }}=1.52$ ) as the material for the replica. Finally, the multilayer coatings were deposited on the base grating, by alternating high and low refractive index thin films.

"Sidrabe" coating system equipped with glancing angle deposition (GLAD) technology was used in all evaporation processes. GLAD method allowed us to modulate the porosity of the thin films by changing the deposition angle between the substrate normal and evaporation flux. This enabled us to vary the refractive index of individual layers easily and with high precision [33]. During the coating process, the corrugated substrates were placed in a holder constantly rotating around its axis to ensure the conformal evaporation. Theoretical modeling indicates that the refractive index difference between high and low index layers for optimal spatial filtering should be around 0.12. To reach the desired refractive index contrast the deposition angles between the substrate normal 
and the vapour flux were $0^{\circ}$ and $65^{\circ}$ for $\mathrm{n}_{\mathrm{H}}=1.42$ and $\mathrm{n}_{\mathrm{L}}=1.30$ respectively. Based on the calculations, the designed structures with $N_{L}=33$ and $N_{L}=39$ layers were deposited starting with the higher refractive index material. The physical thickness of each layer was equal to $120 \mathrm{~nm}$ (value for $d_{z} / 2$ ) and controlled with a quartz crystal monitor. The coating processes were started at room temperature, in high vacuum of $\mathrm{p}_{0}=1.7 \times 10^{-5} \mathrm{mbar}$ and $2 \mathrm{sccm}$ of oxygen gas were introduced into the chamber to ensure the oxidation of evaporated material. The deposition rate was maintained at $3 \AA / s$.

Surface morphology of the grated substrate was measured by Dimension Edge atomic force microscope (AFM) by Bruker. Height distribution measurements of $20 \times 20 \mu^{2}$ surface areas were performed in tapping mode and in ambient conditions. A commercial silicon probe with a tip diameter of $<10 \mathrm{~nm}$ (force constant $-40 \mathrm{~N} / \mathrm{m}$ ) was used.

The inner structure of the samples was characterized using a scanning electron microscope (SEM) workstation Helios Nanolab 650. The cross sections were made by ion beam milling procedure and later analyzed by SEM. The spectroscopic measurements were performed by spectrophotometer Photon RT (Essent Optics). Linearly polarized light was used for two perpendicular polarizations: $\mathrm{S}$ and $\mathrm{P}$, where $\mathrm{S}$ polarization is perpendicular to the grating lines on the substrate. The transmission was measured in the spectral region from $450 \mathrm{~nm}$ to $850 \mathrm{~nm}$ at different angles of incidence. The angle between the mean plane of grating and the detector was changed from $-40^{\circ}$ to $40^{\circ}$ by steps of $2^{\circ}$.

For characterization, we tested the $\mathrm{N}_{\mathrm{L}}=39$ layer structure by probing it with a divergent beam to make sure that the filtering effect can be observed not only for plane waves but also for beams with curved wavefronts. We focused an S-polarized Gaussian beam with a wavelength of $\lambda=532 \mathrm{~nm}$ with a $2.5 \mathrm{~cm}$ focal length cylindrical lens. The beam was characterized by a full divergence angle of $\sim 6^{\circ}$. The transmittance was measured onto the sample which we mounted on a rotating conical mount. The sample was mounted at $21^{\circ}$ angle to the incident beam to approximate an effective wavelength of $\lambda_{\text {eff }}=570 \mathrm{~nm}$. The filtered beam was projected directly on the matrix of a CCD camera at the far-field. The angle between the mean plane of grating and the detector was changed from $-40^{\circ}$ to $40^{\circ}$ by steps of $1^{\circ}$, and the total spatial transmittance spectrum has been averaged for overlapping angular positions.

\subsection{Experimental results}

According to the AFM measurements, the produced blazed substrate pitch was $d_{x}=1.67 \mu \mathrm{m}$ and the peak to valley height was $150 \mathrm{~nm}$ (Fig. 4(a)). After depositing the alternating high and low refractive index layers, the blazed surface gradually changed to a smooth sinusoidal surface, as can be seen in Fig. 4(b) SEM image of the sample crosssection. Although, the surface is changing, the horizontal periodicity $d_{x}$ of the structure remains unchanged. 
(a)
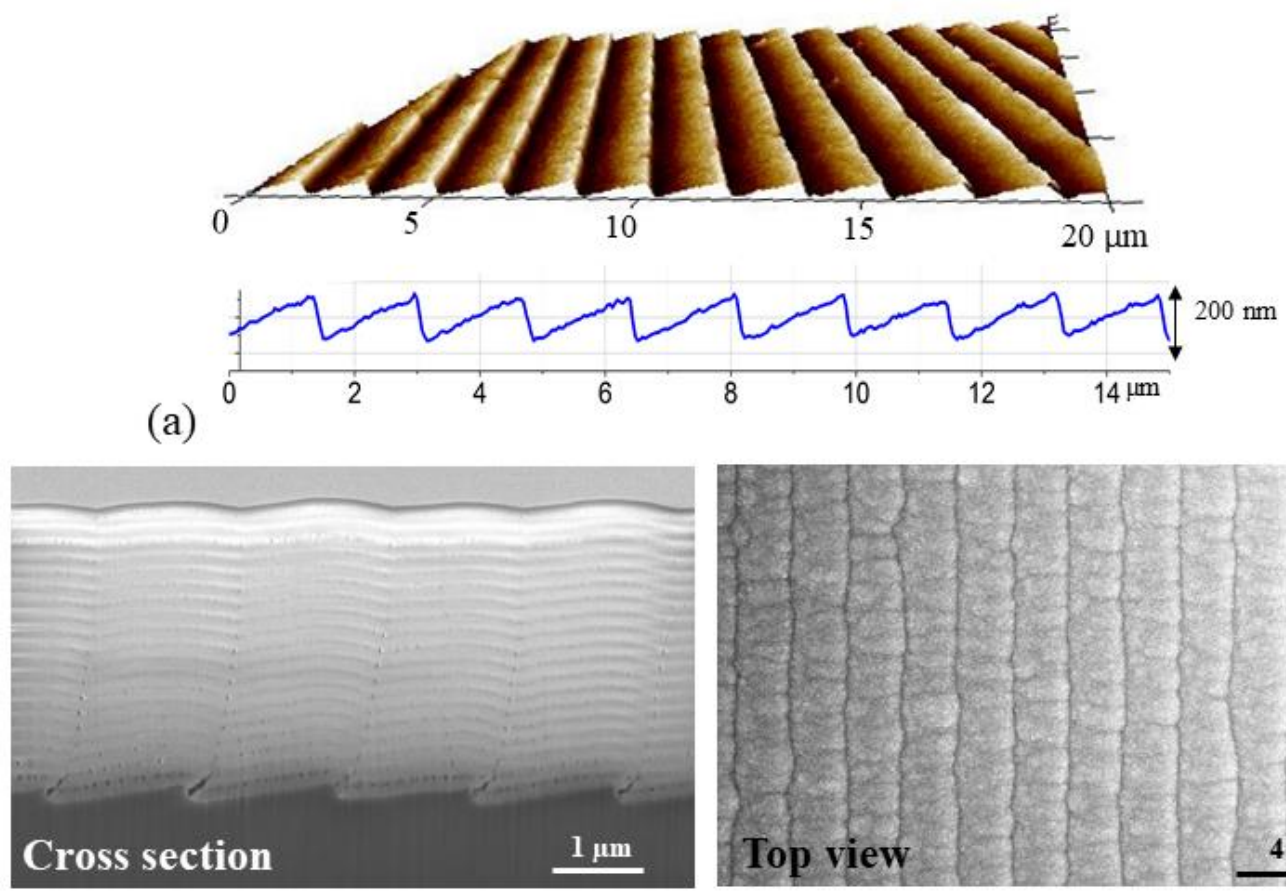

(b)

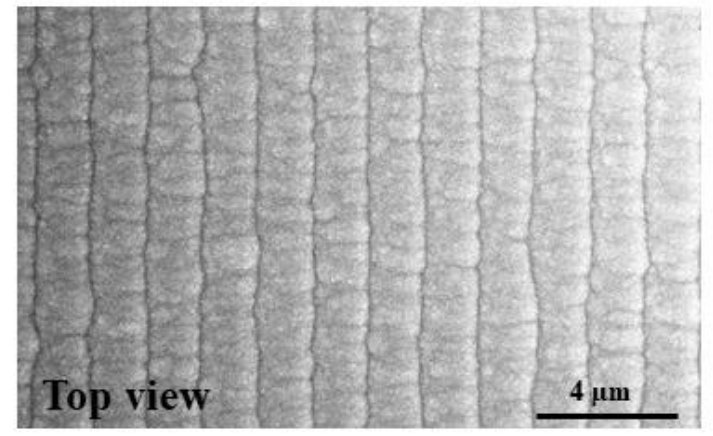

(c)

Figure 4. (a) AFM $3 D$ plot with its height distribution of the corrugated substrate surface. SEM images of fabricated PhC structure (b) cross-section and (c) top view.

The measurements of zero-order angular transmittance profiles are summarized in Fig. 5. In Fig. 5(a) and 5(b) we show the complete transmittance map of the structures having 33 and 39 layers. As expected, the selective decrease in transmittance is more pronounced for the structure with a larger number of longitudinal periods. Also, when comparing the general trend with the numerical results from Fig. 3, we see a qualitative similarity with the predicted results for a harmonic template profile, although there is some asymmetry induced by the blazed profile. Ultimately, the result coincides with superimposed maps from Fig. 3(a) and Fig. 3(b). We interpret this as a result of the continuous change of each individual layer from the blazed to a more harmonic profile (see Fig. 4(b)).

In Fig. 5(c) we see explicit evidence for the hybrid blazed-to-harmonic structure and how much the asymmetry is expressed. For the particular wavelength of $\lambda=572 \mathrm{~nm}, 4$ filtering bands are apparent that correspond roughly to $\pm 15^{\circ}$ and $\pm 28^{\circ}$. The smaller angle filtering band minimum transmittance is $60 \%$. The larger angle resulting from the Bragg condition is asymmetric. Transmittance is reduced to $30 \%$ and $10 \%$, whereas the zeroangle transmittance is at around $80 \%$. Fig. 5(d) shows the result when the sample is probed with a diverging beam. The transmittance profile is even more clearly asymmetric. The bands for positive angles feature transmittance values comparable to the zero-angle component, whereas the positive angle bands are in correspondence to Fig. 5(c). However, all the cases show that we created selective attenuation in a form of photonic band gap operation. 
(a)

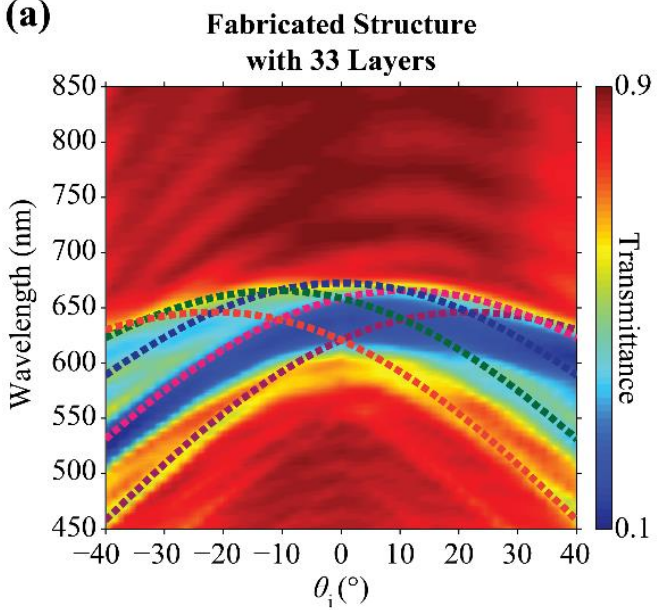

(c)

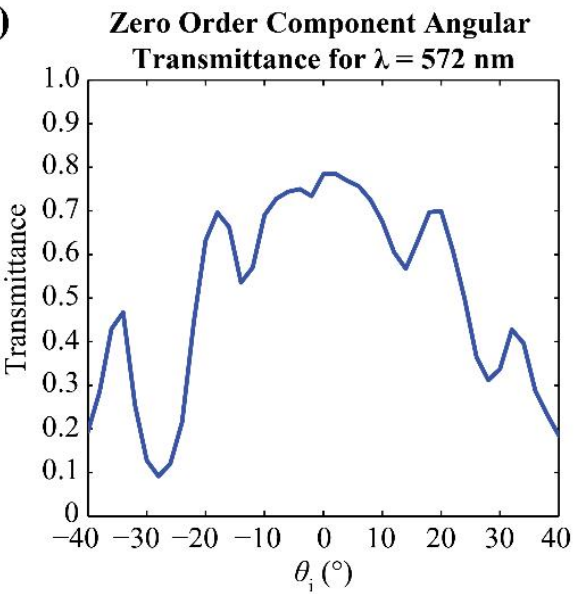

(b)

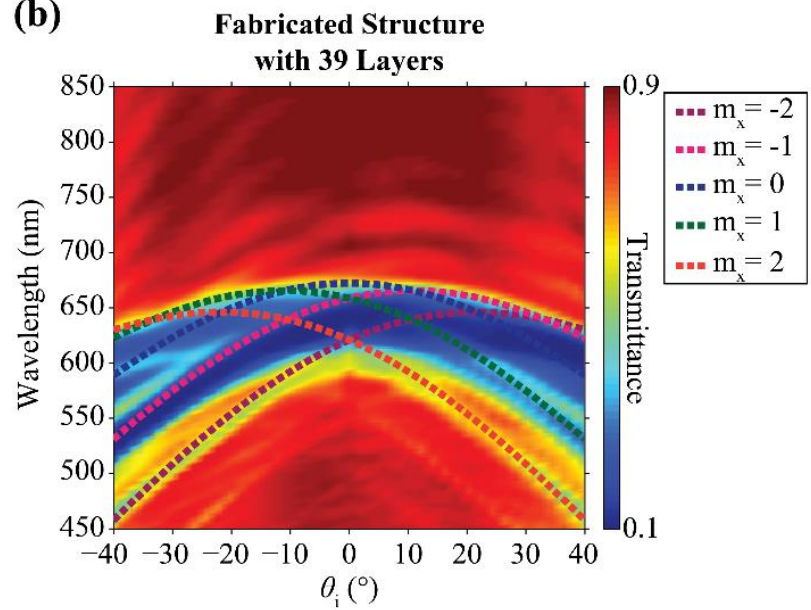

(d) Averaged Diverging Beam Angular

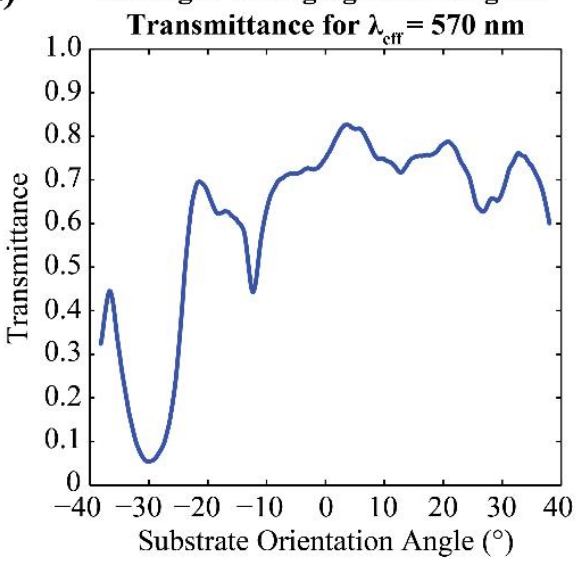

Figure 5. Zero-order spatial transmittance maps measured using a collimated beam for a fabricated $N_{L}=33\left(\right.$ a) and $N_{L}=39$ (b) layer structure. (c) Spatial transmittance profile (horizontal cross-section of the map in $(b)$ ) measured for the fabricated structure with $N_{L}=39$ layers, (d) averaged angular transmittance of a sample at a constant tilt $21^{\circ}$ angle illuminated by a diverging beam with fixed wavelength $\lambda=532 \mathrm{~nm}$, which is equivalent to the normal incidence with an effective wavelength of $\lambda_{\text {eff }}=570 \mathrm{~nm}$. Dashed lines indicate the analytical dependencies according to Eq.(2).

\section{Perspectives}

Filtering performance of the multilayered wavy structure can be improved by further tuning the periods and the contrast of the refractive indexes, which is beyond the current fabrication possibilities. To observe and understand the effect of changing the transverse period $d_{x}$, we performed numeric simulations of wavy structures having structural parameters same as in section 3. In this regard, operating wavelength is fixed as $\lambda=582$ $\mathrm{nm}$ and the zero-order transmission is computed for transverse periods $d_{x}$ between 835 $\mathrm{nm}$ and $3340 \mathrm{~nm}$. Corresponding incidence angle $\theta_{i}$ versus transverse period $d_{x}$ transmission map is given in Fig. 6(a). The angular filtering effect increases with the decrease of the transverse period for the specified wavelength value. For a better comparison, a cross-sectional profile of the transmittance map is calculated at $d_{x}=835 \mathrm{~nm}$ and $d_{x}=564 \mathrm{~nm}$, which are represented in Figs. 6(b) and 6(c). When the 
profiles in Figs. 6(b) and 6(c) are compared with the profile in Fig.3(c) (structure with $d_{x}=1.67 \mu \mathrm{m}$ ), we see that the low-pass transmittance peak (between $-10^{\circ}$ and $10^{\circ}$ ) is getting sharper with the decrease of the transverse period.

Thereafter, the zero-order transmittance angular filtering characteristic was computed for the structures with $d_{x}=835 \mathrm{~nm}$ and $d_{x}=564 \mathrm{~nm}$. Results for varied wavelengths are shown in Fig. 6(d) and 6(g). For both cases, the cross sectional profiles are calculated for selected wavelengths (denoted by black solid lines) and presented in Fig.6. An even narrower transmittance peak is observed at $\lambda=641 \mathrm{~nm}$ for $d_{x}=835 \mathrm{~nm}$. The crosssectional profile is presented in Fig. 6(f). Here, the filtering bands are broader and extend well beyond the incidence angles $\left|\theta_{i}\right|>10^{\circ}$. Hence, for a more pronounced filtering effect that is appropriate for practical applications in the optical regime, the transverse periods should be $\lesssim 2$ times smaller than our experimental test value $d_{x}=1.67 \mu \mathrm{m}$.

(a)

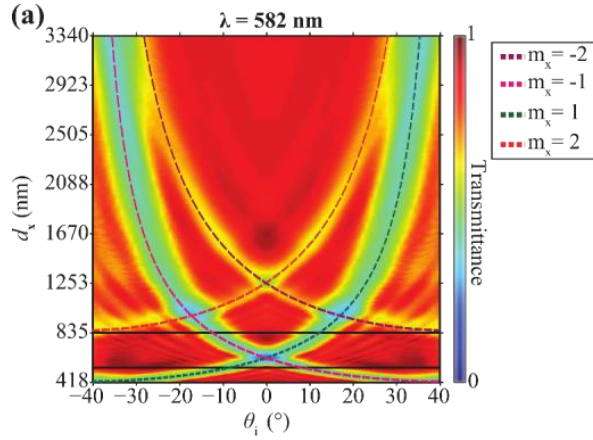

(d)

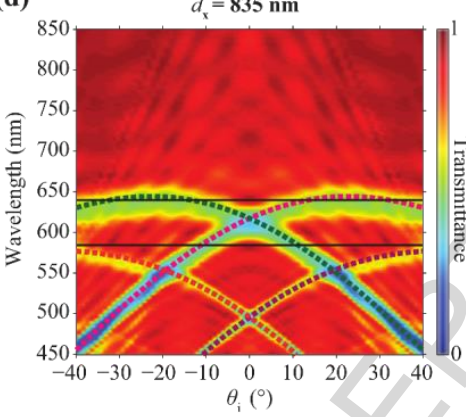

(g)

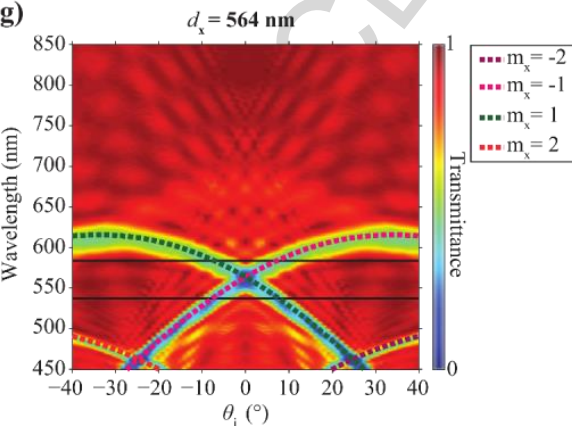

(b)

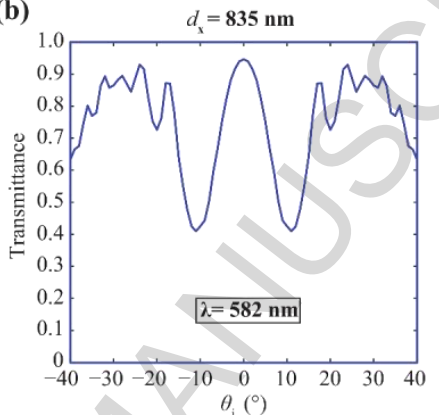

(e)

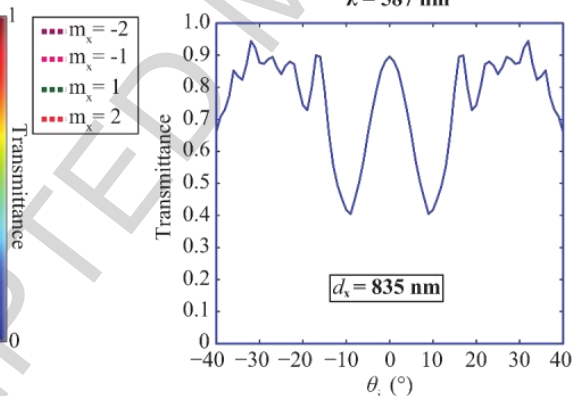

(h)

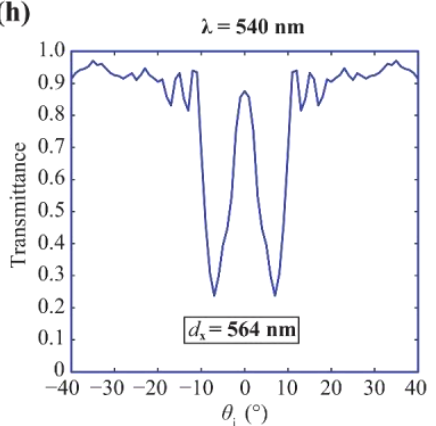

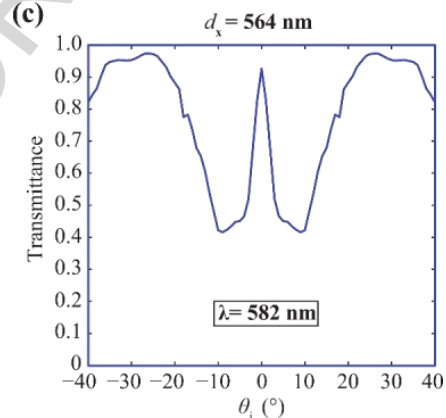

(f)

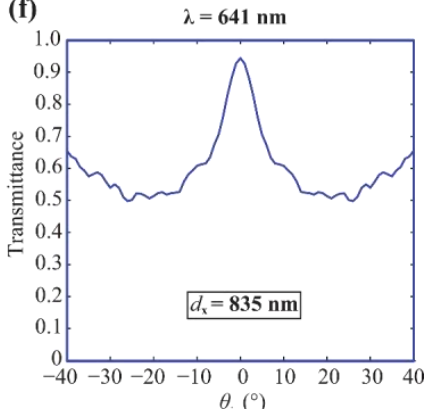

(i)

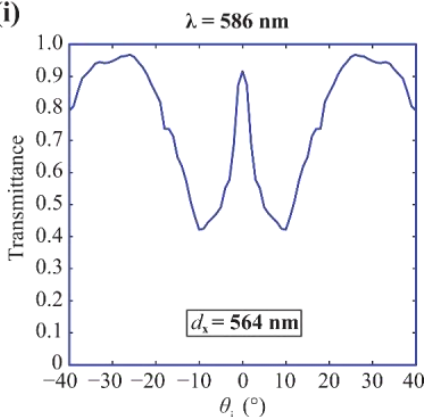

Figure 6. Zero-order angular transmittance maps (a) for the transverse period $d_{x}$, when the probing wavelength $\lambda$ is fixed at $\lambda=582 \mathrm{~nm},(d)$ for $\lambda$, when $d_{x}=835 \mathrm{~nm}$, (c) for $\lambda$, when $d_{x}=564 \mathrm{~nm}$. The middle and right columns show corresponding selected transmittance graphs for (b) $\lambda=582 \mathrm{~nm}$ and $d_{x}=835 \mathrm{~nm}$, (c) $\lambda=582 \mathrm{~nm}$ and $d_{x}=564 \mathrm{~nm}$, (e) $\lambda=587 \mathrm{~nm}$ and $d_{x}=835 \mathrm{~nm}$, (e) $\lambda=641 \mathrm{~nm}$ and $d_{x}=835 \mathrm{~nm},(h) \lambda=540 \mathrm{~nm}$ and $d_{x}=564 \mathrm{~nm}$, (i) $\lambda=586 \mathrm{~nm}$ 
and $d_{x}=564 \mathrm{~nm}$. For all structures: $d_{z}=240 \mathrm{~nm}, n_{H}=1.42, n_{L}=1.33, N_{L}=33$. Dashed lines indicate the analytical dependences, Eg. (2).

\section{Conclusions}

Here, we demonstrated the signatures of spatial filtering in Bragg regime, based on periodic photonic microstructures fabricated by physical vapour deposition. Further developments need to be made for such structures to be of practical use, however the principle of angular filtering has been convincingly demonstrated. The main reason for the relatively weak filtering performance of our structures is the relatively large transverse periods. As evidenced by the Eq. (2), the filtering parabolas for each Bragg component cross at small angles; therefore, the narrow low-angle-pass transmission peaks could not be obtained. In order to show the potential, we present the calculations using the structures beyond the technological fabrication possibilities currently available by us. The master gratings required for these structures can be potentially fabricated using more advanced techniques, such as electron beam lithography. In this regard, the presented results in Section 5 show a promising case and evidences a perspective of practical application of the spatial filters being developed based on the idea promoted in the current article. The presented technology is highly flexible: the transverse period can be controlled from tens of nanometers to micrometers using different methods. Longitudinal period and optical constants can also be tuned precisely with thin film deposition methods. Additionally, the proposed spatial filter can be applied as a coating to any surface, indicating its high suitability to complex microlaser systems.

\section{Acknowledgments:}

K.S acknowledges support by the EUROSTARS Project E!10524 HIP-Lasers, as well as by Spanish Ministerio de Ciencia e Innovación, and European Union FEDER through project FIS2015-65998-C2-1-P. D.G. and V.P. acknowledge the financial support from "FOKRILAS" (Project No. P-MIP-17-190) from Research Council of Lithuania. Authors are grateful to dr. Algirdas Selskis from State research institute Center for Physical Sciences and Technology for SEM measurements.

\section{Declarations of interest: none}

\section{References}

1. K. Staliunas and V. J. Sánchez-Morcillo, "Spatial filtering of light by chirped photonic crystals," Phys. Rev. A 79(5), 053807 (2009).

2. E. Colak, A. O. Cakmak, A. E. Serebryannikov, and E. Ozbay, "Spatial filtering using dielectric photonic crystals at beam-type excitation," J. Appl. Phys. 108(11), 113106 (2010).

3. Z. Luo, Z. Tang, Y. Xiang, H. Luo, and S. Wen, "Polarization-independent lowpass spatial filters based on one-dimensional photonic crystals containing negative-index materials," Appl. Phys. B 94(4), 641-646 (2009). 
4. L. Maigyte, T. Gertus, M. Peckus, J. Trull, C. Cojocaru, V. Sirutkaitis, and K. Staliunas, "Signatures of light-beam spatial filtering in a three-dimensional photonic crystal," Phys. Rev. A 82(4), 043819 (2010).

5. V. Purlys, L. Maigyte, D. Gailevičius, M. Peckus, M. Malinauskas, and K. Staliunas, "Spatial filtering by chirped photonic crystals," Phys. Rev. A 87(3), 033805 (2013).

6. D. Gailevicius, V. Koliadenko, V. Purlys, M. Peckus, V. Taranenko, and K. Staliunas, "Photonic Crystal Microchip Laser," Sci. Rep. 6(1), 34173 (2016).

7. A. A. Skorynin, V. A. Bushuev, and B. I. Mantsyzov, "Dynamical Bragg diffraction of optical pulses in photonic crystals in the Laue geometry: Diffractioninduced splitting, selective compression, and focusing of pulses," J. Exp. Theor. Phys. 115(1), 56-67 (2012).

8. S. E. Svyakhovskiy, A. A. Skorynin, V. A. Bushuev, S. V. Chekalin, V. O. Kompanets, A. I. Maydykovskiy, T. V. Murzina, and B. I. Mantsyzov, "Experimental demonstration of selective compression of femtosecond pulses in the Laue scheme of the dynamical Bragg diffraction in 1D photonic crystals," Opt. Express 22(25), 31002 (2014).

9. H. Y. Ling, Y. Q. Li, and M. Xiao, "Electromagnetically induced grating: Homogeneously broadened medium," Phys. Rev. A - At. Mol. Opt. Phys. 57(2), 1338-1344 (1998).

10. A. W. Brown and M. Xiao, "All-optical switching and routing based on an electromagnetically induced absorption grating," Opt. Lett. 30(7), 699 (2005).

11. L. Zhao, W. Duan, and S. F. Yelin, "All-optical beam control with high speed using image-induced blazed gratings in coherent media," Phys. Rev. A - At. Mol. Opt. Phys. 82(1), 1-8 (2010).

12. R. M. Arkhipov, A. V. Pakhomov, M. V. Arkhipov, I. Babushkin, A. Demircan, U. Morgner, and N. N. Rosanov, "Population density gratings induced by few-cycle optical pulses in a resonant medium," Sci. Rep. 7(1), 1-21 (2017).

13. S. Maruo, O. Nakamura, and S. Kawata, "Three-dimensional microfabrication with two-photon-absorbed photopolymerization," Opt. Lett. 22(2), 132 (1997).

14. G. D. Marshall, M. Ams, and M. J. Withford, "Femtosecond inscription of wavelength specific features in optical waveguide structures," in Integrated Optics, Silicon Photonics, and Photonic Integrated Circuits, G. C. Righini, ed. (2006), 6183, p. 61830Q.

15. M. Malinauskas, A. Žukauskas, S. Hasegawa, Y. Hayasaki, V. Mizeikis, R. Buividas, and S. Juodkazis, "Ultrafast laser processing of materials: from science to industry," Light Sci. Appl. 5(8), e16133 (2016). 
16. M. Campbell, D. N. Sharp, M. T. Harrison, R. G. Denning, and A. J. Turberfield, "Fabrication of photonic crystals for the visible spectrum by holographic lithography," Nature 404(6773), 53-56 (2000).

17. C. K. Ullal, M. Maldovan, E. L. Thomas, G. Chen, Y.-J. Han, and S. Yang, "Photonic crystals through holographic lithography: Simple cubic, diamond-like, and gyroid-like structures," Appl. Phys. Lett. 84(26), 5434-5436 (2004).

18. S. Wilbrandt, O. Stenzel, and N. Kaiser, "Experimental determination of the refractive index profile of rugate filters based on in situ measurements of transmission spectra," J. Phys. D. Appl. Phys. 40(5), 1435-1441 (2007).

19. R. J. Martín-Palma, C. G. Pantano, and A. Lakhtakia, "Replication of fly eyes by the conformal-evaporated-film-by-rotation technique," Nanotechnology 19(35), 355704 (2008).

20. A. Sentenac and A.-L. Fehrembach, "Angular tolerant resonant grating filters under oblique incidence," J. Opt. Soc. Am. A 22(3), 475 (2005).

21. Young Ju Lee, Junho Yeo, R. Mittra, and Wee Sang Park, "Application of electromagnetic bandgap (EBG) superstrates with controllable defects for a class of patch antennas as spatial angular filters," IEEE Trans. Antennas Propag. 53(1), 224-235 (2005).

22. H. lizuka, N. Engheta, and S. Sugiura, "Extremely small wavevector regime in a one-dimensional photonic crystal heterostructure for angular transmission filtering," Opt. Lett. 41(16), 3829 (2016).

23. J. G. Mutitu, Shouyuan Shi, A. Barnett, and D. W. Prather, "Angular Selective Light Filter Based on Photonic Crystals for Photovoltaic Applications," IEEE Photonics J. 2(3), 490-499 (2010).

24. Q. Qian, C. Xu, and C. Wang, "All-dielectric polarization-independent optical angular filter," Sci. Rep. 7(1), 16574 (2017).

25. P. V. Usik, A. E. Serebryannikov, and E. Ozbay, "Spatial and spatial-frequency filtering using one-dimensional graded-index lattices with defects," Opt. Commun. 282(23), 4490-4496 (2009).

26. O. F. Siddiqui and G. V. Eleftheriades, "Resonant modes in continuous metallic grids over ground and related spatial-filtering applications," J. Appl. Phys. 99(8), 083102 (2006).

27. A.-L. Fehrembach, A. Talneau, O. Boyko, F. Lemarchand, and A. Sentenac, "Experimental demonstration of a narrowband, angular tolerant, polarization independent, doubly periodic resonant grating filter," Opt. Lett. 32(15), 2269 (2007).

28. A. Taflove and S. C. Hagness, Computational Electrodynamics (2005). 
29. "Lumerical FDTD Solutions, Inc., http://www.lumerical.com for high performance FDTD-method Maxwell solver for the design, analysis and optimization of nanophotonic devices, processes and materials.," (n.d.).

30. B. Liang, M. Bai, H. Ma, N. Ou, and J. Miao, "Wideband Analysis of Periodic Structures at Oblique Incidence by Material Independent FDTD Algorithm," IEEE Trans. Antennas Propag. 62(1), 354-360 (2014).

31. "Lumerical Inc. https://kb.lumerical.com/en/ref_sim_obj_sources_bfast.html for Broadband Fixed Angle Source Technique (BFAST), which only applies to the periodic structures illuminated with a broadband source at an angle," (n.d.). Retrieved on $11^{\text {th }}$ of February 2019.

32. Y. Xia and G. M. Whitesides, "Soft Lithography," Angew. Chemie Int. Ed. 37(5), 550-575 (1998).

33. T. Tolenis, L. Grinevičiūtè, R. Buzelis, L. Smalakys, E. Pupka, S. Melnikas, A. Selskis, R. Drazdys, and A. Melninkaitis, "Sculptured anti-reflection coatings for high power lasers," Opt. Mater. Express 7(4), 1249 (2017). 
*Angular filtering by photonic crystals in a Bragg configuration was proposed.

*Photonic microstructures were fabricated by physical vapour deposition.

*Angle-wavelength transmission measurements show the signatures of spatial filtering

*FDTD calculations predict that the filtering effect can be substantially enhanced 